sungsmittel restlos. Das geschlossene System erlaubt eine vollständige Rückgewinnung des Lösungsmittels. Als Resultat erhält man einen trockenen, porösen, lösungsmittelfreien Block aus Photoresist.

\section{Funktionsbeschreibung}

Der Destillationsrückstand wird durch eine Schlammpumpe (8) in den Behälter (2), der mit einem Abfallbeutel (3) ausgekleidet ist, gepumpt. Das Fassungsvermögen dieses Behälters beträgt $c a$. 501 . Die Vakuumpumpe (6) setzt das gesamte System unter Vakuum. Der Mikrowellengenerator (7) liefert die erforderliche Energie, um das Lösungsmittel auszudampfen. Das ausgedampfte Lösungsmittel wird im Kondensator (4) kondensiert und in einem Auffangbehälter (5) aufgefangen. Am Ende des Zyklusses schaltet das Gerät automatisch aus. Der erhärtete, trockene, lösungsmittelfreie Rückstand kann auf einfache Weise mit dem Abfallbeutel aus dem SOLIDEX entnommen werden.

Das SOLIDEX-Gerät (Fig. 5 und 6) ist selbstverständlich mit allen erforderlichen Sicherheitsvorrichtungen ausgerüstet, wie z.B.

- einem Infrarotsensor, welcher die Temperatur des Rückstandes im SOLIDEX erfasst und den Mikrowellengenerator ausschaltet, wenn eine vorgegebene Solltemperatur erreicht ist.

- Der Mikrowellengenerator kann nur senden, wenn die Türe geschlossen und das vorgegebene Vakuum vorhanden ist.

Die SOLIDEX-Geräte sind vollautomatisch - ausser der Entnahme des Kuchens am Ende des Zyklusses und dem Einlegen

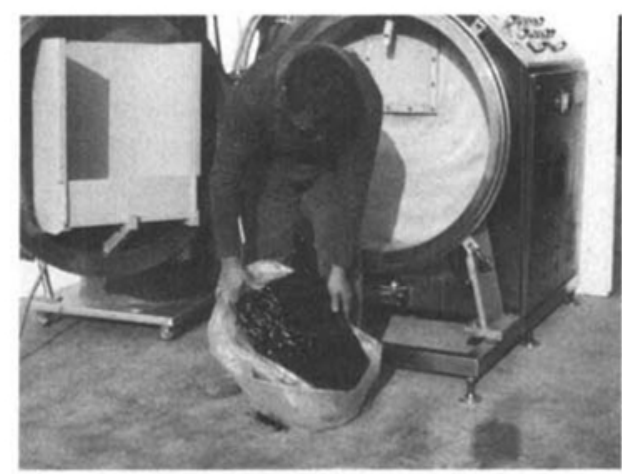

Fig. 6. SOLIDEX (offen)

eines neuen Abfallbeutels. Die produktberührten Teile sind aus Inox oder PP. Man sieht in der Tür den Aufnahmebehälter aus PP. Es ist zu sehen, dass der trockene Rückstand (Fig. 7) auf einfache Weise mit dem Abfallbeutel aus dem Gerät entnommen werden kann. Die gleichmässige, durchgehende, poröse Struktur des trockenen Rückstandes ist ersichtlich. Durch die direkte Einwirkung der Mikrowellen auf das Lösungsmittel trocknet der Rückstand von innen nach aussen und man erhält einen porösen trockenen Kuchen. Diese Trocknungsart erspart aufwendige Kontrollen, denn eine Sichtkontrolle von aussen genügt, um festzustellen, ob der $\mathrm{Ku}$ chen trocken ist. Diese trockenen Kuchen führt man der Verbrennung zu. Es wäre aber ideal, wenn sich eine Applikation finden liesse, bei welcher diese Kuchen zu einem Sekundärrohstoff aufgearbeitet werden könnten.

Das Beispiel in der Tabelle gilt für Destillationsrückstände aus einem Vakuumdestillator. Die Energiekosten für ein SOLIDEX-Gerät sind wie bereits erwähnt, sehr gering - bei $6000 \mathrm{Bh} / \mathrm{Jahr}$ betragen diese nur $c a$. Fr. 700.--/Jahr.

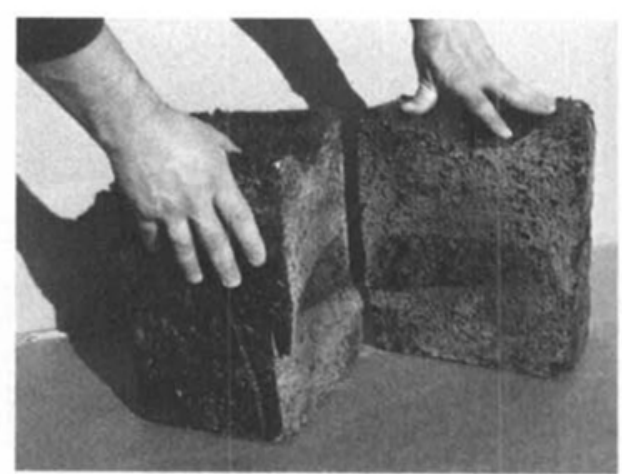

Fig. 7. Trockener Rückstand (Muster)

\section{Wirtschaftlichkeit}

Bei einem Anschaffungspreis eines SOLIDEX von $c a$. Fr. 80000 -- ergibt sich eine Amortisationszeit von weniger als einem Jahr. Ferner ist zu erwähnen, dass nicht nur die Vernichtungskosten, sondern ebenso die Handhabungskosten beträchtlich reduziert werden können, weil anstelle von aufwendigen Fasslagern das Aufstellen einer einfachen Mulde genügt, um die Destillationsrückstände zu lagern, bis diese der Entsorgung zugeführt werden.

\section{Schlussbetrachtung}

Die Rückgewinnung von Lösungsmitteln ist heute ein absolutes MUSS. Das SOLIDEX-Verfahren erlaubt es, nun CKW's auch dort wirtschaftlich zurückzugewinnen, wo konventionelle Verfahren bislang keine befriedigenden Resultate erbringen konnten oder gar gescheitert sind. Neben der Anwendung in der Leiterplattenfertigung sind selbstverständlich noch viele andere Applikationen für dieses Verfahren möglich.

\title{
Umdenken in der Abfallwirtschaft: Volkswirtschaftliche Aspekte des Materialrecycling
}

\author{
Gunter Stephan*
}

Veröffentlichungen zum Thema Abfall vermitteln nicht selten den Eindruck, wir stünden vor einem Müllnotstand. Diesen Eindruck kann ich nicht widerlegen oder verwischen. Dennoch scheint es mir wenig angebracht, in diesem Zusammenhang von einem Notstand zu sprechen. Denn das Wort Notstand suggeriert eine Situation,

\footnotetext{
* Korrespondenz: Prof. Dr. G. Stephan

Abteilung für Angewandte Mikroökonomie

Universität Bern

Gesellschaftsstrasse 27, CH-3012 Bern
}

in der der unmittelbare und massive Eingriff des Staates in die Wirtschaft unvermeidlich ist. Ich dagegen bin der Meinung, dass Abfallprobleme ökologisch und ökonomisch angemessener gelöst werden können, wenn der Staat nicht reglementierend in die Abfallwirtschaft eingreift, sondern Anreize schafft, die zur Vermeidung und Wiederverwertung von Abfällen führt.

Entwickelte Volkswirtschaften stehen heute vor zwei Problemen, deren Lösung sich gegenseitig behindert und scheinbar ausschliesst: Einerseits muss schonend mit der Natur und den knappen Deponieflächen umgegangen werden, um in der $\mathrm{Zu}$ kunft Leben und Wirtschaften zu ermöglichen. Andererseits soll die Innovationskraft der Wirtschaft gefördert werden, um Industriestandorte, Arbeitsplätze und den Wohlstand langfristig zu sichern. Beide Probleme können nur gelöst werden, wenn ein Umdenken in doppelter Hinsicht stattfindet: Erstens müssen bestehende Wirtschaftsstrukturen durch eine weniger roh- 
sungsmittel restlos. Das geschlossene System erlaubt eine vollständige Rückgewinnung des Lösungsmittels. Als Resultat erhält man einen trockenen, porösen, lösungsmittelfreien Block aus Photoresist.

\section{Funktionsbeschreibung}

Der Destillationsrückstand wird durch eine Schlammpumpe (8) in den Behälter (2), der mit einem Abfallbeutel (3) ausgekleidet ist, gepumpt. Das Fassungsvermögen dieses Behälters beträgt $c a$. 501 . Die Vakuumpumpe (6) setzt das gesamte System unter Vakuum. Der Mikrowellengenerator (7) liefert die erforderliche Energie, um das Lösungsmittel auszudampfen. Das ausgedampfte Lösungsmittel wird im Kondensator (4) kondensiert und in einem Auffangbehälter (5) aufgefangen. Am Ende des Zyklusses schaltet das Gerät automatisch aus. Der erhärtete, trockene, lösungsmittelfreie Rückstand kann auf einfache Weise mit dem Abfallbeutel aus dem SOLIDEX entnommen werden.

Das SOLIDEX-Gerät (Fig. 5 und 6) ist selbstverständlich mit allen erforderlichen Sicherheitsvorrichtungen ausgerüstet, wie z.B.

- einem Infrarotsensor, welcher die Temperatur des Rückstandes im SOLIDEX erfasst und den Mikrowellengenerator ausschaltet, wenn eine vorgegebene Solltemperatur erreicht ist.

- Der Mikrowellengenerator kann nur senden, wenn die Türe geschlossen und das vorgegebene Vakuum vorhanden ist.

Die SOLIDEX-Geräte sind vollautomatisch - ausser der Entnahme des Kuchens am Ende des Zyklusses und dem Einlegen

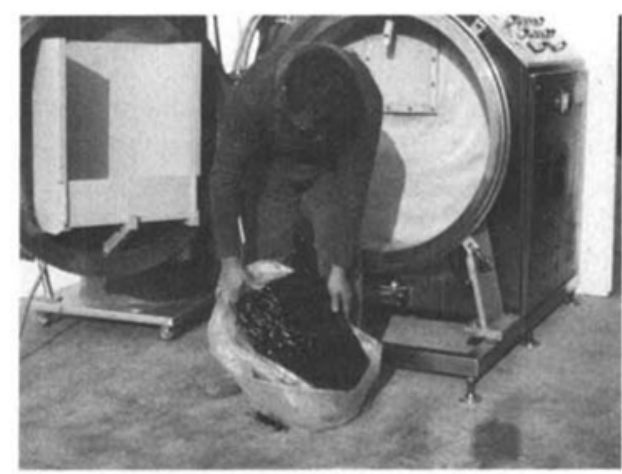

Fig. 6. SOLIDEX (offen)

eines neuen Abfallbeutels. Die produktberührten Teile sind aus Inox oder PP. Man sieht in der Tür den Aufnahmebehälter aus PP. Es ist zu sehen, dass der trockene Rückstand (Fig. 7) auf einfache Weise mit dem Abfallbeutel aus dem Gerät entnommen werden kann. Die gleichmässige, durchgehende, poröse Struktur des trockenen Rückstandes ist ersichtlich. Durch die direkte Einwirkung der Mikrowellen auf das Lösungsmittel trocknet der Rückstand von innen nach aussen und man erhält einen porösen trockenen Kuchen. Diese Trocknungsart erspart aufwendige Kontrollen, denn eine Sichtkontrolle von aussen genügt, um festzustellen, ob der $\mathrm{Ku}$ chen trocken ist. Diese trockenen Kuchen führt man der Verbrennung zu. Es wäre aber ideal, wenn sich eine Applikation finden liesse, bei welcher diese Kuchen zu einem Sekundärrohstoff aufgearbeitet werden könnten.

Das Beispiel in der Tabelle gilt für Destillationsrückstände aus einem Vakuumdestillator. Die Energiekosten für ein SOLIDEX-Gerät sind wie bereits erwähnt, sehr gering - bei $6000 \mathrm{Bh} / \mathrm{Jahr}$ betragen diese nur $c a$. Fr. 700.--/Jahr.

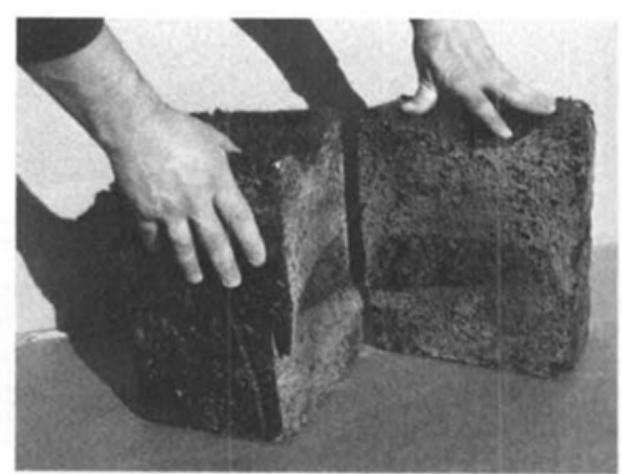

Fig. 7. Trockener Rückstand (Muster)

\section{Wirtschaftlichkeit}

Bei einem Anschaffungspreis eines SOLIDEX von $c a$. Fr. 80000 -- ergibt sich eine Amortisationszeit von weniger als einem Jahr. Ferner ist zu erwähnen, dass nicht nur die Vernichtungskosten, sondern ebenso die Handhabungskosten beträchtlich reduziert werden können, weil anstelle von aufwendigen Fasslagern das Aufstellen einer einfachen Mulde genügt, um die Destillationsrückstände zu lagern, bis diese der Entsorgung zugeführt werden.

\section{Schlussbetrachtung}

Die Rückgewinnung von Lösungsmitteln ist heute ein absolutes MUSS. Das SOLIDEX-Verfahren erlaubt es, nun CKW's auch dort wirtschaftlich zurückzugewinnen, wo konventionelle Verfahren bislang keine befriedigenden Resultate erbringen konnten oder gar gescheitert sind. Neben der Anwendung in der Leiterplattenfertigung sind selbstverständlich noch viele andere Applikationen für dieses Verfahren möglich.

\title{
Umdenken in der Abfallwirtschaft: Volkswirtschaftliche Aspekte des Materialrecycling
}

\author{
Gunter Stephan*
}

Veröffentlichungen zum Thema Abfall vermitteln nicht selten den Eindruck, wir stünden vor einem Müllnotstand. Diesen Eindruck kann ich nicht widerlegen oder verwischen. Dennoch scheint es mir wenig angebracht, in diesem Zusammenhang von einem Notstand zu sprechen. Denn das Wort Notstand suggeriert eine Situation,

\footnotetext{
* Korrespondenz: Prof. Dr. G. Stephan

Abteilung für Angewandte Mikroökonomie

Universität Bern

Gesellschaftsstrasse 27, CH-3012 Bern
}

in der der unmittelbare und massive Eingriff des Staates in die Wirtschaft unvermeidlich ist. Ich dagegen bin der Meinung, dass Abfallprobleme ökologisch und ökonomisch angemessener gelöst werden können, wenn der Staat nicht reglementierend in die Abfallwirtschaft eingreift, sondern Anreize schafft, die zur Vermeidung und Wiederverwertung von Abfällen führt.

Entwickelte Volkswirtschaften stehen heute vor zwei Problemen, deren Lösung sich gegenseitig behindert und scheinbar ausschliesst: Einerseits muss schonend mit der Natur und den knappen Deponieflächen umgegangen werden, um in der $\mathrm{Zu}$ kunft Leben und Wirtschaften zu ermöglichen. Andererseits soll die Innovationskraft der Wirtschaft gefördert werden, um Industriestandorte, Arbeitsplätze und den Wohlstand langfristig zu sichern. Beide Probleme können nur gelöst werden, wenn ein Umdenken in doppelter Hinsicht stattfindet: Erstens müssen bestehende Wirtschaftsstrukturen durch eine weniger roh- 


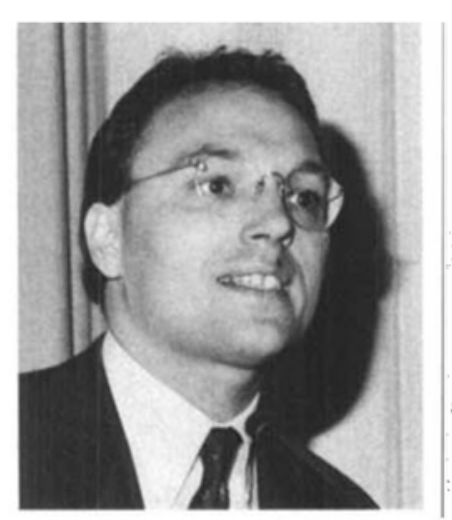

Gunter Stephan: Geboren am 12. August 1952 in Eberbach, Rhein-Neckar-Kreis, BRD 1959 Einschulung in die Grundschule, Eberbach. 1971 Abitur am Hohenstaufen-Gymnasium Eberbach. 1971-1976 Studium der Mathematik, Physik und Volkswirtschaftslehre, Universität Heidelberg. 1976-1977 Prüfung zum Diplommathematiker 1980 Promotion zum Dr. rer. pol. 1979-1983 Mitarbeit im DFG Projekt: Umwelt- und Ressourcenökonomie, Heidelberg. 1983-1984 Forschungsaufenthalt, Stanford University, USA. 1986 Forschungsaufenthalt, Statistical Bureau, Norway. 1987 Habilitation, Wirtschaftswissenschaftliche Fakultät, Universität Heidelberg, Ernennung zum Privatdozenten. 1987-1988 Gutachter für das Umweltministerium Baden-Württemberg und Mitglied der LAGA (ständige Kommission der Bundesländer über Abfälle). 1988 Beförderung zum Hochschuldozenten. Oktober 1988 Extraordinanus für Volkswirtschaftslehre, Universität Bern.

stoff- und emissionsintensive Wirtschaftsweise abgelöst werden. Zweitens muss die heute übliche Praxis, Umweltpolitik über staatliche Verordnungen durchzuführen, durch flexible und kostengünstige Instrumente ersetzt werden, die Anreize schaffen, den Rohstoffverbrauch zu verringern, Emissionen und Abfälle zu vermeiden oder wenigstens wiederzuverwerten

Recycling ist eine Möglichkeit, den Rohstoffverbrauch und das Abfallaufkommen einer Wirtschaft insgesamt zu verringern. Recycling zielt jedoch nicht auf die Vermeidung $a b$, sondern umfasst Verfahren, die - einer bestehenden Konsum- und Produktionsstruktur nachgeschaltet - Wertstoffe aus Abfällen wiedergewinnen. Recycling setzt damit keine technologische Ânderung in der Produktions- und Konsumweise voraus. Recyclingverfahren werden vielmehr einer bestehenden Struktur nachgeschaltet und können deshalb bereits kurzfristig zur Entspannung der Rohstoffund Abfallproblematik beitragen.

Trotz dieser Vorzüge wird nur ein geringer Teil der technisch verwertbaren Abfälle dem Recycling zugeführt. Es wird geschätzt (s. [1]), dass es technisch möglich ist, mehr als $35 \%$ aller Abfälle wiederaufzubereiten. Das heute tatsächlich erreichte Recyclingvolumen erfasst im Mittel aber nur ca. $15 \%$ (vgl. [2]). Ein Grund für den geringen Umfang an Wiederverwertung ist, dass Recycling in vielen Fällen betriebswirtschaftlich zu teuer ist. Natürlich kann man erwarten, dass mit der Verbesserung der Verfahren auch deren Rentabilität steigt. Nach meinem Dafürhalten reicht dies jedoch nicht aus, um Recycling konkurrenzfähig zu machen. Vielmehr muss sich die Bewertung von Gütern und Emissionen über die Märkte ändern.

In einer Marktwirtschaft lenken Preise die Entscheidungen der Unternehmungen und Haushalte. In einer idealen Marktwirtschaft trägt dabei das wirtschaftliche Interesse des Einzelnen zum Wohl aller bei, und die Summe der betriebswirtschaftlichen Kosten entspricht der Summe der volkswirtschaftlichen Aufwendungen zur Bereitstellung von Gütern und Dienstleistungen. Dabei erfüllen die Preise zwei zentrale Funktionen: 1. Preise geben die Knappheit an Gütern und Dienstleistungen wieder: Je höher das Bedürfnis nach einem Gut, und je weniger von einem Gut vorhanden ist, desto höher ist der Preis dieses Gutes. 2. Preise haben eine Kompensationsfunktion. Der Marktpreis muss in einer idealen Marktwirtschaft alle Kosten abdecken, die bei der Bereitstellung von Gütern entstehen. Dies umfasst die Kosten für alle Importfaktoren, aber auch die Kosten der Entsorgung von Emissionen. In einer idealen Marktwirtschaft wird damit das Verursacherprinzip in dem Sinne durchgesetzt, dass der Preis eines Gutes einen monetären Gegenwert zu allen Aufwendungen und Nachteilen darstellt, die andern durch die Bereitstellung oder den Konsum dieses Gutes entstehen.

Im Fall von Rohstoff- und Umweltproblemen sind diese beiden Funktionen von Preisen und damit der Markt in seiner Funktion gestört. Am Beispiel der Weltmarktpreise für fossile Rohstoffe wird dies sofort deutlich. In der Realität sind die Preise von Rohstoffen im wesentlichen durch die betriebswirtschaftlichen Kosten der Förderung und des Transportes bestimmt. Nicht berücksichtigt werden in den Preisen von Ressourcen in der Regel die fortschreitende Verknappung von Rohstoffen und die Kosten, die einer Volkswirtschaft entstehen, nachdem ein Rohstoff erschöpft ist und auf einen anderen oder auf andere Technologien ausgewichen werden muss.

Ähnliches gilt für die Kosten der Abfallbehandlung und Umweltbelastung, die bei der Förderung von Rohstoffen, deren Verwendung in der Produktion sowie späteren Verbrauch der Produkte entstehen. Die Folgen von Umweltbelastungen, wie beispielsweise die Entsorgung von Altlasten, werden meist nicht vollständig vom Verursacher, sondern teilweise von der Gemeinschaft getragen. Sie gehen ebensowenig in die Güterpreise ein wie die Tatsache, dass Güter, die heute produziert und konsumiert werden, morgen zu Abfall werden, der kostbaren Deponieraum verbraucht und damit zur Verknappung von Deponieflächen, somit zum Müllnotstand beitragen. (Für eine ausführlichere Diskussion s. [3].)

Mit anderen Worten: Im Falle von Umwelt- und Ressourcenproblemen sind zwe zentrale Funktionen von Preisen gestört. Die auf den Märkten beobachteten Preise von Ressourcen oder die Gebühren für die Inanspruchnahme von Deponieflächen ge- ben nicht die tatsächliche Knappheit wieder und setzen nicht das Verursacherprinzip um. Im Gegenteil, sie sind in der Regel viel zu tief. Von ihnen gehen deshalb die falschen Signale aus, und nur ein Teil der tatsächlichen volkswirtschaftlichen Kosten zur Herstellung von Gütern wird über Preise an die einzelnen Betriebe weitergegeben: Die betriebs- und volkswirtschaftlichen Kosten weichen so weit voneinander ab, dass es aus volkswirtschaftlicher Sicht zu einer Vergeudung von Rohstoffen und einer Schädigung, ja sogar Zerstörung der Umwelt kommt.

Diese Form von Marktversagen, die durch die Fehlfunktion von Preisen hervorgerufen wird und zur Asymmetrie zwischen betriebs- und volkswirtschaftlichen Kosten führt, hat Auswirkungen auf das Recycling. Wie ich schon angedeutet habe, ist in vielen Fällen Recycling betriebswirtschaftlich nicht rentabel und Recyclingprodukte können mit vergleichbaren, aber direkt aus originären Rohstoffen hergestellten Produkten nicht konkurrieren. Denn beim Recycling werden ja Wertstoffe aus Abfällen und anderen Emissionen gewonnen. Deshalb geht ein Teil der volkswirtschaftlichen Umwelt- und Abfallkosten in die Betriebskosten von Recycling ein, die bei der Produktion aus originären Ressourcen zwar auch anfallen, dort aber auf Grund der angesprochenen Fehlfunktion des Marktes nicht von Produzenten, sondern von der Gemeinschaft getragen werden.

Wie kann in dieser Situation Recycling forciert werden? Die ökonomische Theorie besagt, dass im Falle von Marktversagen staatliche Eingriffe notwendig sind. Prinzipiell stehen dem Staat dabei zwei Alternativen zur Verfügung: Er kann erstens über Gebote, Verbote und Auflagen den verstärkten Einsatz, von Recycling bindend vorschreiben; oder er kann zweitens durch Abgaben die Preise von Gütern auf ihre volkswirtschaftlich 'richtige' Höhe korrigieren und die Fehlfunktion des Marktes beheben.

Aus volkswirtschaftlicher Sicht ist die zweite Massnahme vorzuziehen. Durch staatliche Vorschriften wird Recycling ja nicht betriebswirtschaftlich rentabel. Das heisst, Recycling muss in irgendeiner Form von aussen (ausserhalb der Unternehmungen) gestützt und finanziert werden, was die Gefahr beinhaltet, dass öffentliche Stellen Recycling subventionieren, betreiben und die technischen Standards prägen. Ebenso werden keine Signale gesetzt, die das Eigeninteresse der Bürger zu verstärktem Recycling wecken und die Entwicklung neuer kostengünstiger Verfahren anregen. Im Gegenteil, ökonomisches Handeln der Betroffenen wird durch den Zwang der Vorschriften behindert. Denn über Gebote und Verbote versucht der Staat ja direkt und lenkend wie in einer Planwirtschaft in den Wirtschaftsprozess einzugreifen.

Abgaben dagegen schreiben Entsorgungswege nicht bindend vor, sondern 
schaffen durch eine Korrektur der Preise Anreize ohne den Handlungsspielraum des Einzelnen einzuengen. Denn eine solche Korrektur erhöht ausschliesslich die in die betriebswirtschaftliche Kalkulation eingehenden Preise, was dynamische Anpassungsreaktionen auslöst: Erstens steigen aufgrund von Marktkräften über die Zeit auch die Preise von Recyclingprodukten. Damit kommt Recycling verstärkt zum Einsatz und die gewonnenen Güter können auch vermarktet werden. Zweitens beschleunigt der erhöhte Preisdruck die Einführung weniger rohstoff- und emissionsintensiver Produktionstechniken und Recyclingverfahren, die bei den heutigen Preisen noch nicht rentabel betrieben werden können. Drittens käme es zu technischen Erfindungen, was die Kostenrelation zwischen Primär- und Sekundärrohstoffen zusätzlich zugunsten des Recyclingsektors verbessert. Letzteres ist wichtig, denn moderner Umweltschutz benötigt die Nutzung technischer Innovation. (Für eine weitergehende Betrachtung s. [2].)

Trotz der genannten wirtschaftstheoretischen Vorzüge einer Abfallabgabe wäre es naiv anzunehmen, dass kurzfristig Abgaben auf Rohstoffe und Abfälle eingeführt werden und in der Abfallwirtschaft ausschliesslich marktkonforme Instrumente zum Tragen kommen. Puristische Lösungen sind vielleicht theoretisch interessant, haben aber in der Regel wenig Chancen auf eine Umsetzung. Widerstände gegen abfallwirtschaftliche Regulierungen, die ausschliesslich auf Lenkungsabgaben setzen, sind aus verschiedenen gesellschaftlichen Gruppen zu erwarten: Unternehmen, die Abfälle produzieren, Verwaltungsfachleute, die mit der Durchführung umweltpolitischer Gesetze befasst sind, Politiker, die Gesetze zwar beschliessen, aber dem Wahlverfahren unterworfen sind. Realistischerweise wird man daher erwarten, dass umweltpolitische Reglementierungen einen Kompromiss zwischen den Vorstellungen der am politischen Prozess Beteiligten darstellen und eine Mischung aus einer Auflagen- und Abgabenpolitik vorsehen.

Aber auch eine kombinierte Auflagenund Abgabenlösung wird sich nicht von heute auf morgen verwirklichen lassen. Dennoch kann der Staat kurzfristig Recycling fördern, ohne dirigistische Instrumente einsetzen zu müssen. Die 'flankierenden' Massnahmen umfassen: 1. Die Unterstützung der Erforschung, Entwicklung und des Ausbaus von Recycling durch zinsgünstige Kredite. Hierzu gehört auch, dass die Behörden kleine und mittlere Firmen, die keine eigenen Erfahrungen im Recycling haben, technisch beraten und Unternehmungen wie Haushalte über Vermeidungs- und Recyclingmöglichkeiten aufklären. 2. Die Erhöhung der Abfallbeseitigungsgebühren, um die traditionelle Abfallbeseitigung durch Deponierung zugunsten des Recycling zu verteuern, was im Prinzip einer Abfallabgabe entsprechen würde. 3. Die Stützung von Recyclingmärkten in der Phase ihrer Entstehung.
Unter einer globalen Perspektive ist zu erwarten, dass eine zunehmende Recyclingtätigkeit zumindest mittelfristig zu Verringerungen des Rohstoffverbrauchs, der Umweltschädigungen, der Abfallmengen und damit zur Schonung von knappen Deponievolumen führt.

Für tiefergehende Aussagen der ökonomischen Probleme, die mit dem Recycling verbunden sind, müssen die verschiedenen Recyclingverfahren zumindest in drei $\mathrm{Ka}$ tegorien unterteilt werden (s. [4]): 1. primäres Recycling, worunter die innerbetriebliche Weiterverwendung verstanden ist; 2. sekundäres Recycling, das die Verwertung bei Dritten anspricht, und 3. tertiäres Recycling, die Verwertung nach privatem Konsum.

Primäres Recycling ist oft technisch komplex und investitionsintensiv. Sind solche Verfahren jedoch installiert, dann ist ihr Einsatz relativ problemlos. Denn das Ausgangsmaterial wird von der Firma selber geliefert und das Recyclingprodukt kann innerbetrieblich wieder verwendet werden. Damit ist es der Firma möglich, Recycling relativ unabhängig von temporären Marktschwankungen zu betreiben.

Diese Situation ist beim sekundären und tertiären Recycling nicht gegeben. In diesen beiden Fällen müssen Märkte errichtet und gesichert werden, auf denen Ausgangs- und/oder Endprodukte des Recyclings gehandelt werden. Damit ist sowohl der Preis für das Ausgangsmaterial als auch für das Recyclingprodukt abhängig vom Marktgeschehen und wird insbesondere von den Preisschwankungen auf den anderen Güter- und Weltrohstoffmärkten beeinflusst, was Unsicherheit verursacht. Zusätzlich unterliegt sekundäres Recycling dynamischen Anpassungs- und Veränderungsreaktionen. Mit verbesserten und weniger abfallintensiven Technologien ist damit zu rechnen, dass die Menge des verfügbaren Ausgangsmaterials für das Recycling abnimmt. Beispielsweise werden heute noch viele Umweltschutzmassnahmen in Form einer nachsorgenden Schadstoffminderung durchgeführt. Dabei fallen grosse Abfallmengen an, die prinzipiell dem Recycling zugeführt werden können. Langfristig muss aber die End-of-the-Pipe-Entsorgung aufgegeben und zum integrierten Umweltschutz mit weniger umweltschädigenden Produktionsverfahren (Clean Technologies) übergegangen werden, womit das Abfallaufkommen sinkt. Berücksichtigt man, dass der Aufbau und die eventuelle Umstellung einer Recyclingbranche nicht unbeträchtlicher Zeiträume bedarf, so erkennt man, wie stark sekundäres Recycling mit Unsicherheit verbunden ist.

Darüberhinaus besteht beim tertiären Recycling, der Wiederverwertung von Hausmüll, die Notwendigkeit, Abfälle mit aufwendigen Sammel-, Transport- und/ oder Trennsystemen vom Verbraucher zum Verwerter zu bringen. Im Einzelfall könnte der Nettoeffekt der Recyclingtätigkeit sogar negativ sein und forciertes Re- cycling den Transport gefährlicher Stoffe ausdehnen.

Ungeachtet der insgesamt positiven Bewertung und der mittelfristig absehbaren Entspannung der Müll- und Abfallproblematik muss aber festgehalten werden, dass Recycling nur eine nachsorgende Entsorgungsstrategie ist. Recycling zielt nicht auf die Vermeidung von Emissionen und $\mathrm{Ab}$ fällen $a b$, sondern bedeutet nur eine teilweise und nachsorgende Wiederverwertung von Wertstoffen aus Abfällen. Eine staatliche Förderung muss langfristig aber stets berücksichtigen, dass die Vermeidung und Verringerung von Emissionen und Abfällen den Vorrang vor der Verwertung hat. Aus gesamtwirtschaftlicher Sicht trägt die Innovation emissionsarmer Technologien stärker zur Rohstoffeinsparung und Umweltentlastung bei als Recycling; insbesondere auch deshalb, weil 1. nicht alle Abfallarten recyclierbar sind, 2. eine vollständige Rückgewinnung der in Abfällen enthaltenen Rohstoffe aufgrund physikalischer Gesetzmässigkeiten nicht möglich ist, und 3. Abfälle nicht beliebig oft recycliert werden können. Damit verlängert Recycling nur die Verweildauer von Stoffen im wirtschaftlichen Kreislauf. Letztlich aber werden sie dennoch zu Abfall, der beseitigt werden muss. Damit führt Verwertung zwar zu einer mittelfristigen Entspannung der Rohstoff- und Abfallproblematik, beinhaltet aber keine langfristige Lösungsstrategie. Im Gegenteil, eine überzogene staatliche Förderung von Recycling kann die Einführung neuer Technologien zur Vermeidung von Abfällen verzögern.

Fassen wir zusammen: Der Markt ist aus sich heraus nicht in der Lage, Umweltund Ressourcenprobleme zu lösen. Daraus folgt nicht, dass der Staat über Gebote und Verbote direkt lenkend in die Wirtschaft eingreifen muss. Vielmehr kann eine Lösung über den Markt besser sein als jede andere, sofern angemessene Signale durch Abgaben gegeben werden. Soll die Stärke des Marktes ausgenutzt werden, muss langfristig eine Vorwärtsstrategie eingesetzt werden: Statt einer einseitig an Auflagen orientierten Umweltpolitik müssen flexible und kostengünstige Instrumente verwendet werden, die marktwirtschaftliche Anreize schaffen, Emissionen zu vermeiden und Abfälle zu verwerten. Statt vorwiegend Umweltreparaturen von oben zu verordnen, muss beim Verursacher problemgerechter Umweltschutz gefördert werden. Dies gilt auch für das Recycling. Recycling ist nur dort einzusetzen, wo eine Vermeidung nicht absehbar ist.

[1] R. Nolte, 'Mengen- und wertmässiges Recyclingpotential in der BRD', Entsorga Schriften 2, 1985.

[2] M. Faber, G. Stephan, P. Michaelis, 'Umdenken in der Abfallwirtschaft', 2. Aufl., Springer-Verlag, Heidelberg-Berlin, 1989.

[3] G. Stephan, 'Umweltschutz, eine ökologische, politische und technische Aufgabe', 'Journal für Oberfläche und Technik 12/87', 1987.

[4] M. Faber, G. Stephan, 'Volkswirtschaftliche Betrachtungen zum Materialrecycling', Müll und Abfall $3 / 89,1989$. 